\title{
Impacts des changements climatique et environnemental sur la réponse hydrologique du bassin du Nakanbé à Wayen (Burkina Faso) à travers le cadre de budyko
}

\author{
Yetchékpo Patrick Gbohoui ${ }^{1,2}$, Jean-Emmanuel Paturel ${ }^{2}$, Tazen Fowe ${ }^{1}$, Harouna Karambiri ${ }^{1}$, and \\ Hamma Yacouba ${ }^{1}$ \\ ${ }^{1}$ Laboratoire Eaux, HydroSystèmes et Agriculture (LEHSA), Institut International d'Ingénierie de l'Eau et de \\ l'Environnement (2iE), Rue de la Science, Ouagadougou 01 01-BP-594, Burkina Faso \\ ${ }^{2}$ HydroSciences Montpellier, Univ Montpellier, CNRS, IRD, 163 rue Auguste Broussonnet, \\ 34090, Montpellier, France \\ Correspondence: Y. Patrick Gbohoui (patrickgbohoui@gmail.com)
}

Published: 16 November 2021

Résumé. Les changements climatique et environnemental ont entraîné l'augmentation exacerbée des coefficients d'écoulement dans certains hydrosystèmes du Sahel ouest africain. Ce "paradoxe hydrologique sahélien » a fait l'objet de plusieurs études, mais très peu d'entre elles ont abordé les contributions de chacun des changements climatique et environnemental. L'objectif de cette étude est de quantifier les contributions de chacun des facteurs (climat et environnement) au changement des écoulements dans la partie sahélienne du bassin du Nakanbé sur la période 1965-1994. Les tests statistiques de stationnarité ont permis de découper la période d'étude en sous-périodes de base et d'impact. La quantification des impacts a été effectuée à travers l'application des techniques d'élasticité et de décomposition aux modèles Budyko de Fu et de Choudhury. L'analyse des chroniques de données hydro-climatiques du bassin a confirmé le paradoxe hydrologique avec une rupture dans les écoulements en 1977 (test de Pettitt, $p$ value=0,021). Ainsi, la période 1978-1994 comparativement à 1965-1977 a été caractérisée par une diminution des précipitations de 8,2\%, une augmentation de l'évapotranspiration potentielle de $1,3 \%$ et une augmentation des écoulements de $91,5 \%$. Il ressort de cette étude que les contributions à l'augmentation des écoulements de $91,5 \%$ ont été de $-29 \pm 2 \%$ pour le changement climatique, $+90 \pm 1 \%$ pour la dégradation environnementale et $+33 \%$ pour les interactions couplées climat-environnement. Ces résultats montrent que l'impact de l'environnement est plus prépondérant et représente environ 3 fois celui du climat en intensité. L'adoption de meilleures pratiques de gestion de la dynamique environnementale pourrait donc permettre de réguler les changements dans les écoulements du bassin.

Abstract. Climate and environmental changes have caused the increasing runoff coefficients of some sahelian catchments in West Africa. Many previous studies have focused on this "sahelian hydrological paradox", but few have addressed the individual contribution of climate and the environment. This study aims to quantify the contributions of each factor (climate and environment) to the change of runoff in the sahelian part of the Nakanbé catchment over the period : 1965-1994. Based on time-series stationarity statistical tests, the study period was divided into baseline and impacted periods. The quantification of impacts was carried out by applying elasticity and decomposition techniques to the Budyko type models of $\mathrm{Fu}$ and Choudhury. Statistical analysis of the annual hydro-climatic data detected 1977 as the year of break in the runoff (Pettitt test, $p$ value $=0.021$ ) and confirmed the sahelian hydrological paradox. Thus, the period 1978-1994 compared to 1965-1977 was characterized by a decrease of $8.2 \%$ in precipitation while increases of $1.3 \%$ and $91.5 \%$ have been observed respectively for potential evapotranspiration and annual runoff. The results indicated that the contributions to the runoff increase of $91.5 \%$ were $-29 \pm 2 \%$ for climate change, $+90 \pm 1 \%$ for environmental degradation 
and $+33 \%$ for interactions. It appears that, environmental change is the main cause of the increase in the runoff coefficient in the sahelian catchment of Nakanbé and the intensity of its impact is approximately 3 times more than the one induced by climate change. Therefore, good management of environmental dynamics at the catchment scale could help to regulate runoff changes.

\section{Introduction}

Le changement climatique et la dégradation de l'environnement constituent les causes de changements dans les écoulements. Dans certains hydrosystèmes du Sahel ouest africain, ils se traduisent par une augmentation exacerbée des coefficients d'écoulement. Cet accroissement du coefficient d'écoulement (communément appelé «paradoxe hydrologique sahélien »), constaté par plusieurs auteurs dont une revue a été faite par Descroix et al. (2018), résulterait d'un profond changement du comportement hydrologique de ces hydrosystèmes. La quantification de l'impact de chacune des causes s'avère donc indispensable pour expliquer le paradoxe et en tenir compte dans l'élaboration et/ou l'exécution des politiques d'aménagement, de gestion et de protection des ressources hydriques.

Dans la littérature, les méthodes utilisées pour estimer les impacts peuvent être regroupées en des approches descendante et ascendante (Blöschl et al., 2007). L'approche ascendante (encore appelée approche Newtonienne) englobe l'utilisation des modèles physiques distribués/semi distribués qui permettent les contrôles de causalité, mais les résultats des simulations sont en grande partie le reflet des nombreux paramètres. L'approche descendante (encore appelée approche Darwinienne) concernent les études statistiques/bayésiennes sur de longues séries de données hydro-climatiques et environnementales, les méthodes conceptuelles (cadre de Budyko, cadre de Tomer-Schiling) et les méthodes analytiques (élasticité du climat, sensibilité hydrologique). L'approche ascendante nécessite beaucoup de données difficilement disponibles en quantité et en qualité dont celles de la dynamique environnementale. Les méthodes analytiques et conceptuelles basées sur le cadre conceptuel de Budyko ne nécessitent pas de données environnementales, mais produisent des résultats pratiques utiles et utilisables par les décideurs (Hasan et al., 2018).

Le manque de données fiables de suivi de la dynamique environnementale en Afrique a limité la plupart des études d'impacts uniquement à la quantification des impacts du changement climatique ou à des études qualitatives (Boubacar, 2012; Grijsen et al., 2013; Fowe et al., 2012). Les approches descendantes constituent donc une alternative fiable pour quantifier les impacts réels des changements climatique et environnemental sur la ressource en eau de l'Afrique et surtout du Sahel dont le comportement hydrologique est très fragile.

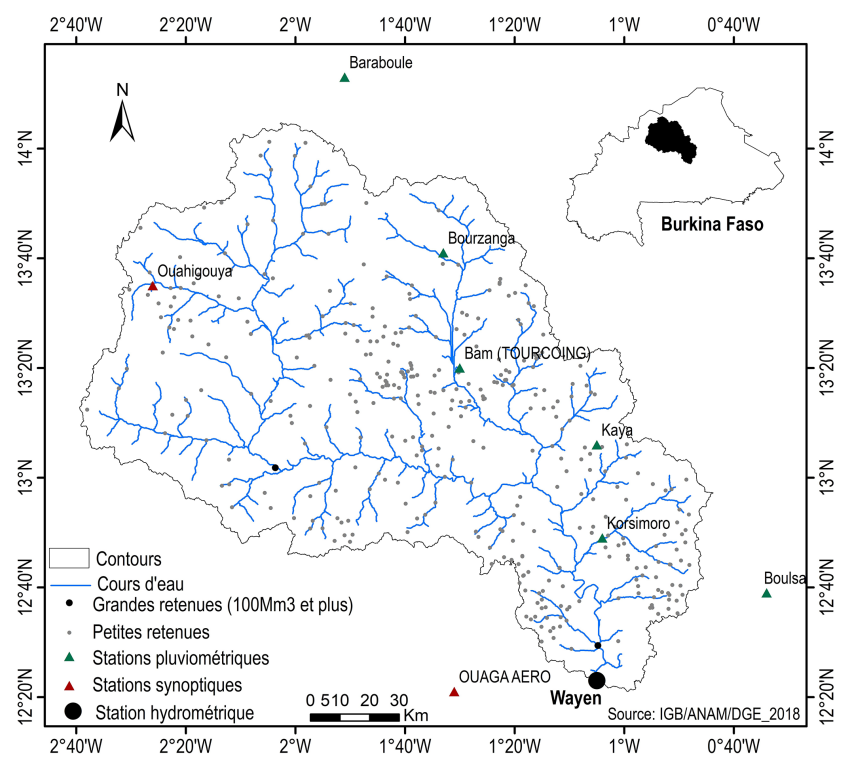

Figure 1. Zone d'étude : Bassin du Nakanbé à Wayen.

L'objectif principale de cette étude est de quantifier les contributions de chacun des changements climatique et environnemental au changement des écoulements dans la partie sahélienne du bassin du Nakanbé au Burkina Faso. Pour ce faire, des tests statistiques de détection de tendance et de rupture ont été utilisés pour choisir les périodes de référence et d'impact. Les impacts ont été quantifiés à travers deux approches et deux modèles, soit de quatre manières différentes.

\section{Données et méthodes}

\subsection{Zone et données de l'étude}

La zone d'étude est la partie sahélienne du bassin du Nakanbé, le bassin du Nakanbé à Wayen qui draine une superficie de $21178 \mathrm{~km}^{2}$ (Fig. 1). Ce bassin est très anthropisé et abrite 371 retenues d'eau (Fig. 1) dont les plus importantes (Ziga et Dourou) ont été mises en eau entre 1995 et 2000. La Fig. 1 montre la position des stations hydro-climatiques utilisées. Les données d'écoulements $(R)$, de précipitations $(P)$ et d'évapotranspirations potentielles $(\mathrm{PE})$ utilisées proviennent des services météorologique et hydrologique du Burkina Faso et de la base SIEREM (Boyer et al., 2006; http://www.hydrosciences.fr/SIEREM/, la date du dernier accès : 20 October 2021). Elles concernent huit stations de pluie, deux synoptiques et une hydrométrique (Fig. 1). L'éva- 
potranspiration réelle (AE) est déduite du bilan hydrique à long terme du bassin en négligeant les échanges avec les nappes souterraines. La pluie moyenne du bassin versant a été calculée en appliquant la méthode du polygone de Thiessen aux huits stations pluviométriques. L'évapotranspiration potentielle du bassin versant a été obtenue en faisant une moyenne pondérée des données aux deux stations synoptiques. L'analyse des données disponibles à chaque station a conduit au choix de la période 1965-1994 pour l'étude. Le choix de cette période se justifie par le fait qu'elle couvre la période de sécheresse (1970-1989) au cours de laquelle le paradoxe hydrologique sahélien a été mis en évidence et qu'elle n'intègre pas la période de mise en eau des grands aménagements sur le bassin (la capacité des deux grands aménagements faisant plus de $80 \%$ du volume de toutes les retenues). Les autres retenues sont supposées intégrées au relief du bassin, car elles sont de petites capacités et beaucoup ont été réalisées autour des années 1960. Aussi, la longueur de cette période est suffisante pour une étude d'impacts (Dey et Mishra, 2017).

\subsection{Cadre conceptuel de Budyko et modèles Budyko adaptés à la zone d'étude}

Le cadre de Budyko (Eq. 1) est une approche basée sur le bilan hydrique et est utilisé par plusieurs auteurs à travers le monde pour quantifier les contributions du climat et de l'environnement au changement des écoulements. Une synthèse de ces travaux a été faite par Dey et Mishra (2017).

$$
\frac{\mathrm{AE}}{P}=f(\varphi, e)=f
$$

où $\mathrm{AE}$ est l'évapotranspiration réelle; $P$ la précipitation; $\varphi$ est le rapport entre l'évapotranspiration potentielle (ETP) et $P ; e$ est un paramètre qui prend en compte l'état de l'environnement du bassin et $f$ est la fonction Budyko.

L'analyse de sensibilité des modèles de type Budyko avait montré que les modèles de Fu (1981) et de Choudhury (1999) sont les plus adaptés au contexte sahélien où les indices d'aridité sont supérieurs à 2 (Gbohoui et al., 2021). Ces deux modèles sont utilisés dans cette étude car les indices d'aridités du bassin ont varié entre 2 et 5 sur la période 1965-1994.

\subsection{Méthodologie de quantification d'impacts}

\subsubsection{Approche d'élasticité améliorée}

En s'inspirant de Gbohoui et al. (2021), les expressions des contributions du changement climatique (Eq. 2), du changement environnemental (Eq. 3) et de l'interaction climatenvironnement (Eq. 4) au changement d'écoulement par rapport à l'écoulement sur la période de référence ont été obte-

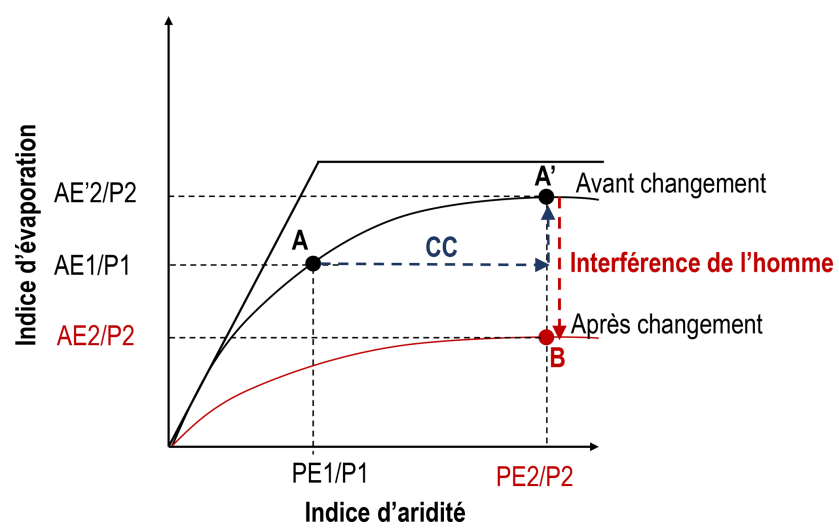

Figure 2. Décomposition de la courbe de Budyko. CC : Changement climatique; AE : Evapotranspiration réelle; PE : Evapotranspiration potentielle; $P$ : Précipitation.

nues.

$$
\begin{aligned}
& \frac{\Delta R_{\mathrm{C}}}{R}=(1+\beta) \frac{\Delta P}{P}-\beta \frac{\Delta \mathrm{PE}}{\mathrm{PE}} ; \beta=\frac{\varphi}{1-f(\varphi, e)} \frac{\partial f}{\partial \varphi} \\
& \frac{\Delta R_{\mathrm{e}}}{R}=\left[\frac{-e}{1-f(\varphi, e)} \frac{\partial f}{\partial e}\right] \frac{\Delta e}{e} \\
& \frac{\Delta R_{\text {ice }}}{R}=\frac{\Delta R}{R}-\frac{\Delta R_{\mathrm{C}}}{R}-\frac{\Delta R_{\mathrm{e}}}{R}
\end{aligned}
$$

où $\frac{\Delta P}{P}, \frac{\Delta \mathrm{PE}}{\mathrm{PE}}, \frac{\Delta R}{R}$ sont les taux de variations de la précipitation, de l'évapotranspiration potentielle et de l'écoulement de la période d'impact par rapport à la période de référence. $\frac{\Delta R_{\mathrm{C}}}{R}, \frac{\Delta R_{\mathrm{e}}}{R}, \frac{\Delta R_{\text {ice }}}{R}$ sont les contributions respectives du climat, de l'environnement et de l'interaction climat-environnement à $\frac{\Delta R}{R}$.

\subsubsection{Approche de décomposition}

La décomposition de la courbe de Budyko est une approche conceptuelle qui se base sur le fait qu'à l'échelle d'un bassin versant, tous les termes (AE, $P$ et PE) du bilan hydrique sont influencés par le climat alors que la dynamique environnementale n'impacte que l'évapotranspiration réelle (AE). La courbe de Budyko étant une relation entre l'indice d'évaporation $(\mathrm{AE} / P$ ) et l'indice d'aridité $(\mathrm{PE} / P)$, si les changements intervenus sur le bassin ne sont dus qu'au climat, sa position bougera mais restera sur la même courbe (ex : A à $\mathrm{A}^{\prime}$ sur la Fig. 2). Tout changement de courbe indique qu'il y a eu d'autres modifications que le changement climatique, donc qu'il y a eu un impact environnemental. L'équation (5) permet de quantifier les variations d'écoulement dues à l'environnement et au climat sans dissocier l'interaction climat-environnement.

$\Delta R_{\mathrm{E}}=P_{2}\left(\frac{\mathrm{AE}_{2}^{\prime}}{P_{2}}-\frac{\mathrm{AE}_{2}}{P_{2}}\right)$ 
Tableau 1. Impacts du climat et de l'environnement sur les écoulements du Nakanbé à Wayen.

\begin{tabular}{|c|c|c|c|c|c|c|c|c|c|}
\hline \multirow[t]{2}{*}{ Période d'impact } & \multirow[t]{2}{*}{$\frac{\Delta P}{P}$} & \multirow[t]{2}{*}{$\frac{\Delta \mathrm{PE}}{\mathrm{PE}}$} & \multirow[t]{2}{*}{$\frac{\Delta R}{R}$} & \multirow[t]{2}{*}{ Approches/Modèles } & \multicolumn{3}{|c|}{ Elasticité } & \multicolumn{2}{|c|}{ Décomposition } \\
\hline & & & & & $\frac{\Delta R_{\mathrm{c}}}{R}$ & $\frac{\Delta R_{\mathrm{e}}}{R}$ & $\frac{\Delta R_{\text {ice }}}{R}$ & $\frac{\Delta R_{\mathrm{c}}}{R}$ & $\frac{\Delta \mathrm{RE}}{R}$ \\
\hline 1978-1994 & $-8,2 \%$ & $1,3 \%$ & $91,5 \%$ & $\begin{array}{l}\text { Fu } \\
\text { Choudhury }\end{array}$ & $\begin{array}{l}-30,6 \% \\
-32,0 \%\end{array}$ & $\begin{array}{l}89,3 \% \\
90,4 \%\end{array}$ & $\begin{array}{l}32,7 \% \\
33,0 \%\end{array}$ & $\begin{array}{l}-27,2 \% \\
-28,3 \%\end{array}$ & $\begin{array}{l}118,7 \% \\
119,8 \%\end{array}$ \\
\hline
\end{tabular}

$\frac{\Delta P}{P}, \frac{\Delta \mathrm{PE}}{\mathrm{PE}}, \frac{\Delta R}{R}$ sont les taux de variations de chacune des variables par rapport à la période de référence : 1965-1977. $\frac{\Delta R_{\mathrm{c}}}{R}, \frac{\Delta R_{\mathrm{e}}}{R}, \frac{\Delta R_{\mathrm{ice}}}{R}$ sont les contributions respectives du climat, de l'environnement et de l'interaction climat-environnement à $\frac{\Delta R}{R}$. Sur la période de référence, les moyennes annuelles des variables hydro-climatiques sont de 636,30/2189,76/9,89 mm respectivement pour la précipitation, l'évapotranspiration potentielle et la lame d'eau écoulée. De 1965 à 1994 , les paramètres environnementaux sont passés de 3,38 ( $w$ de Fu) et 2,58 ( $n$ de Choudhury) sur la période de référence à respectivement 2,79 et 2,01 sur la période d'impact $1978-1994$.

et

$\Delta R_{\mathrm{C}}=P_{2}\left(1-\frac{\mathrm{AE}_{2}^{\prime}}{P_{2}}\right)-R_{1}$

$R_{1}$ désigne la lame d'eau moyenne annuelle écoulée sur la période de base, $\Delta R_{\mathrm{C}}$ et $\Delta R_{\mathrm{E}}$ sont les variations d'écoulement dues respectivement aux changements climatique et environnemental.

\section{Résultats et discussion}

\subsection{Confirmation du paradoxe hydrologique sahélien dans le bassin du Nakanbé à Wayen}

Les tests de détection de tendance de Mann-Kendall et de rupture de Pettitt ont été utilisés pour analyser les séries de données de précipitation, d'évapotranspiration potentielle et d'écoulement sur la période 1965-1994. Les résultats de l'étude de tendance ont montré une augmentation des écoulements annuels (Pente de $\operatorname{Sen}=0,547 ; p$ value $=0,005$ ) et de l'évapotranspiration potentielle (Pente de $\operatorname{Sen}=1,44$; $p$ value $=0,014$ ) alors que les précipitations annuelles du bassin sont en décroissance (Pente de Sen $=-4,17$; $p$ value $=0,256)$. Ce résultat confirme le premier paradoxe hydrologique sahélien (Descroix et al., 2018) caractérisé par une augmentation exacerbée du ruissellement malgré la sécheresse de 1970-1989 et le réchauffement climatique. Le test de Pettitt a indiqué une rupture significative dans les écoulements à l'exutoire du bassin à partir de 1977 ( $p$ value $=0,021$ ). Ainsi, la période 1965-1977 a été considérée comme la période de référence pour les études d'impacts.

\subsection{Impacts des changements climatique et environnemental sur les écoulements du Nakanbé à Wayen}

Nous avons quantifié les impacts des changements climatique et environnemental subis par le bassin sur la période 1978-1994 par rapport à 1965-1977 en utilisant les modèles Budyko de $\mathrm{Fu}$ et de Choudhury. Les résultats (Tableau 1) montrent que :
- L'approche d'élasticité permet de déduire l'impact des interactions environnement-climat;

- Les contributions des différents facteurs à l'accroissement des écoulements de 91,5\% sur la période 19781994 par rapport à la période 1965-1977 ont été de $-29 \pm 2 \%$ pour le climat, $+90 \pm 1 \%$ pour l'environnement et $+33 \%$ pour les interactions climatenvironnement;

- L'impact de l'environnement quantifié selon l'approche de décomposition $(\Delta \mathrm{RE} / R)$ inclus les interactions environnement-climat et a été considéré comme l'impact brut de l'environnement;

- Les deux modèles donnent des résultats similaires : le modèle de Fu surestime les impacts de 1 à $5 \%$ par rapport au modèle de Choudhury;

- L'approche d'élasticité surestime les impacts de 2 à $13 \%$ par rapport à celle de décomposition.

Ces résultats confirment que le premier paradoxe hydrologique mis en évidence est principalement dû à la dégradation environnementale (Descroix et al., 2018) et ont montré une dégradation des paramètres environnementaux de $20 \%$ sur la période 1978-1994 par rapport à 1965-1977. L'adoption de meilleures pratiques de gestion environnementale permettra de réduire les coefficients de ruissellement car il existe une relation consistante et significative entre le taux de couverture végétale et le paramètre des modèles Budyko (Gbohoui et al., 2021 ; Li et al., 2013). Les résultats obtenus sont similaires à ceux de Gbohoui et al. (2021) à l'échelle du Nakanbé à Wayen, malgré le peu de stations utilisées dans la présente étude. Cela montre la robustesse des approches utilisées.

\section{Conclusion}

Dans cette étude, nous avons quantifié les contributions du climat et de l'environnement au premier paradoxe hydrologique sahélien dans le bassin du Nakanbé à Wayen au Burkina Faso sur la base de huit stations de pluie, deux d'évapotranspiration potentiel et d'une station hydrométrique. Nos 
résultats ont montré que l'intensité de l'impact de l'environnement fait plus du triple de celui du climat. L'adoption de meilleures pratiques de gestion de la dynamique environnementale du bassin pourrait donc permettre de réguler les écoulements.

Disponibilité du code. Le code utilisé dans cet article est disponible à https://doi.org/10.5281/zenodo.5590923 (Gbohoui, 2021).

Disponibilité des données. Les données utilisées sont la propriété des organismes nationaux du Burkina Faso : Direction Générale des Ressources en Eau (DGRE), Agence Nationale de la Météorologie (ANAM).

Collaborateurs. YPG a fait la conceptualisation, le traitement et l'analyse des données; élaboré la méthodologie et le code informatique de calcul; rédigé et révisé l'article.

JEP, TF, HK et HY ont participé à la conceptualisation, à l'élaboration de la méthodologie, à l'analyse des résultats et à la rédaction de l'article, et ont supervisé les activités de recherche.

Intérêts concurrents. Les auteurs déclarent qu'ils n'ont aucun conflit d'intérêts.

Clause de non-responsabilité. Publisher's note : Copernicus Publications remains neutral with regard to jurisdictional claims in published maps and institutional affiliations.

Déclaration du numéro spécial. This article is part of the special issue "Hydrology of Large River Basins of Africa". It is a result of the 4th International Conference on the "Hydrology of the Great Rivers of Africa”, Cotonou, Benin, 13-20 November 2021.

Remerciements. Les auteurs remercient toutes les personnes qui ont contribué à cet article en lisant ou en donnant de judicieux conseils.

Financement. This research has been supported by the African Development Bank Group (Project no. P-Z1-IA0- 013, Grant no. 2100155032824).

\section{Références}

Blöschl, G., Ardoin-Bardin, S., Bonell, M., Dorninger, M., Goodrich, D., Gutknecht, D., Matamoros, D., Merz, B., Shand, P., and Szolgay, J. : At what scales do climate variability and land cover change impact on flooding and low flows ?, Hydrol. Process., 21, 1241-1247, https://doi.org/10.1002/hyp.6669, 2007.
Boubacar, I. : Caractérisation des saisons de pluies au Burkina Faso dans un contexte de changement climatique et évaluation des impacts hydrologiques sur le bassin du Nakanbé, Thèse de doctorat, 2iE et Université Pierre et Marie Curie, France, 2012.

Boyer, J.-F., Dieulin, C., Rouche, N., Cres, A., Servat, E., Paturel, J.-E., and Mahe, G. : SIEREM : an environmental information system for water resources, IAHS publication, 308, 19-25, 2006.

Choudhury, B. J. : Evaluation of an empirical equation for annual evaporation using field observations and results from a biophysical model, J. Hydrol., 216, 99-110, 1999.

Descroix, L., Guichard, F., Grippa, M., Lambert, L., Panthou, G., Mahé, G., Gal, L., Dardel, C., Quantin, G., Kergoat, L., Bouaïta, Y., Hiernaux, P., Vischel, T., Pellarin, T., Faty, B., Wilcox, C., Malam Abdou, M., Mamadou, I., Vandervaere, J.-P., DiongueNiang, A., Ndiaye, O., Sané, Y., Dacosta, H., Gosset, M., Cassé, C., Sultan, B., Barry, A., Amogu, O., Nka Nnomo, B., Barry, A., and Paturel, J.-E. : Evolution of Surface Hydrology in the Sahelo-Sudanian Strip : An Updated Review, Water, 10, 748, https://doi.org/10.3390/w10060748, 2018.

Dey, P. and Mishra, A. : Separating the impacts of climate change and human activities on streamflow : A review of methodologies and critical assumptions, J. Hydrol., 548, 278-290, https://doi.org/10.1016/j.jhydrol.2017.03.014, 2017.

Fowe, T., Yacouba, H., Karambiri, H., Barbier, B., Paturel, J.-E., et Boubacar, I. : Impacts des scenarii climatique et de l'occupation des sols sur les ressources en eau du bassin versant du Nakambé (Burkina Faso), Climat et Développement, 14, 123-137, 2012.

$\mathrm{Fu}, \mathrm{B}$. : On the calculation of the evaporation from land surface, Sci. Atmos., 5, 23-31, 1981.

Gbohoui, Y. P. : R script for impacts quantification based on Budyko framework (Version V0). Hydrology of Large River Basins of Africa, Cotonou, Bénin, Zenodo [code], https://doi.org/10.5281/zenodo.5590923, 2021.

Gbohoui, Y. P., Paturel, J.-E., Tazen, F., Mounirou, L. A., Yonaba, R., Karambiri, H., and Yacouba, H. Impacts of climate and environmental changes on water resources : A multi-scale study based on Nakanbé nested watersheds in West African Sahel, J. Hydrol. Reg. Stud., 35, 100828, https://doi.org/10.1016/j.ejrh.2021.100828, 2021.

Grijsen, J. G., Brown, C., Tarhule, A., Ghile, Y. B., Taner, Ü., TalbiJordan, A., Doffou, H. N., Guero, A., Dessouassi, R. Y., Kone, S., Coulibaly, B., and Harshadeep, N. : Climate Risk Assessment for Water Resources Development in theNiger River Basin PartI : Context and Climate Projections, Climate Variability : Regional and Thematic Patterns, 37, BoD-Books on Demand, https://doi.org/10.5772/56707, 2013.

Hasan, E., Tarhule, A., Kirstetter, P.-E., Clark III, R., and Hong, Y. : Runoff sensitivity to climate change in the Nile River Basin, J. Hydrol., 561, 312-321, https://doi.org/10.1016/j.jhydrol.2018.04.004, 2018.

Li, D., Pan, M., Cong, Z., Zhang, L., and Wood, E. : Vegetation control on water and energy balance within the Budyko framework, Water Resour. Res., 49, 969-976, https://doi.org/10.1002/wrcr.20107, 2013. 